\title{
Viabilidade econômica em povoamento de Eucalyptus spp. mal conduzido na Paraíba
}

\author{
Tamires Leal de Lima ${ }^{*}$, Regis Villanova Longhi ${ }^{2}$, Anderson Francisco da Silva ${ }^{1}$, Nivandilmo Luiz da
} Silva $^{2}$, Anderson Arthur Lima dos Santos², Gessyca Fernanda de Sena Oliveira ${ }^{1}$

RESUMO: O objetivo deste trabalho foi avaliar a rentabilidade e produtividade de um povoamento de Eucalyptus spp. mal conduzido no Estado da Paraíba em relação à um povoamento em condições adequadas de manejo e produtividade. Os dados foram obtidos do inventário florestal realizado no povoamento de Eucalyptus spp. com área de 43,7 ha e idade de 10 anos. Esse povoamento teve um baixo investimento de implantação e manutenção, fato que resultou em uma alta taxa de mortalidade, sendo classificado como mal conduzido (Cenário 1). Para quantificação da perda econômica e de produtividade utilizou-se como parâmetro os dados de um povoamento considerado ideal (Cenário 2), sendo aquele em que houve maior investimento para a implantação e manutenção em todas as fases de desenvolvimento, atingindo assim a produtividade esperada para a região do estudo. A viabilidade econômica foi avaliada pelos indicadores econômicos: Valor Presente Líquido (VPL), Taxa Interna de Retorno (TIR) e Relação Benefício/Custo (B/C). Constatou-se que a atividade da eucaliptocultura no Estado da Paraíba é economicamente viável com VPL positivo, TIR maior que a TMA e B/C acima de 1 para ambos os cenários. Porém, o povoamento atual (Cenário 1) apresentou perda de $\mathrm{R} \$ 2.256,43$ ha ${ }^{-1}$ em comparação com o VPL do povoamento esperado. Conclui-se que o povoamento atual deve ser reformado visando um maior retorno econômico.

Palavras-chave: manejo florestal, análise econômica, silvicultura.

\section{Economic viability and productivity of a Eucalyptus spp. poorly managed in the State of Paraíba}

\begin{abstract}
The objective of this work was to evaluate the economic viability and productivity of a poorly managed Eucalyptus stand in the state of Paraíba in relation to a stand with adequate management and productivity conditions. The data were obtained from the forest inventory in the Eucalyptus stand with an area of 43.7 ha and age of 10 years. This stand had a low investment of implantation and maintenance, fact that resulted in a high mortality rate, being classified as poorly managed (Scenario 1). In order to quantify the economic and productivity loss, the data of an ideal stand (Scenario 2) were used as parameter, being that in which there was greater investment for the implantation and maintenance in all the phases of development, thus reaching the expected wood productivity for the study region. The economic viability was evaluated through economic indicators: Net Present Value (NPV), Internal Rate of Return (IRR) and Benefit / Cost Ratio (B/C). We found that eucalyptus activity in the State of Paraíba is economically viable with positive NPV, higher TIR than TMA and B/C higher than 1 for both scenarios. However, the current stand (Scenario 1) presented a loss of R $2,256.43 \mathrm{ha}^{-1}$ compared to the NPV of the expected stand. It is concluded that the current stand must be reformed for a higher economic return.
\end{abstract}

Keywords: forest manegement, economic analysis, forestry.

\section{Introdução}

O aumento do interesse do mercado consumidor por madeira de Eucalyptus spp. é justificada pela multifuncionalidade do produto, por ser uma matéria prima utilizada nas indústrias de celulose, papel, moveleira, ceramista, dentre outras, cujo aumento está diretamente ligado à procura de produtos sustentáveis (MOLEDO et al., 2016). Associado a isso, a eucaliptocultura é desejada do ponto de vista ambiental por ser uma espécie cultivada que não há restrições legais para sua exploração e por ser uma

Recebido em 05/10/2019, Aceito para publicação em 15/10/2019

${ }^{1}$ Universidade Federal Rural de Pernambuco

${ }^{2}$ Universidade Federal de Alagoas

*e-mail: tamires_leal22@hotmail.com espécie adaptada às condições geoambientais da maior parte do território brasileiro.

A atividade da silvicultura é então a saída para minimizar a pressão existente sobre a flora nativa, pois o cultivo de espécies arbóreas requer um manejo adequado para que atividade atinja seu objetivo principal, que é o máximo de produtividade e retorno financeiro. $O$ manejo florestal para eucaliptos por sua vez, envolve não somente as tomadas de decisão para exploração, mas sim, para que ao fim do ciclo o projeto desenvolvido resulte em madeira de qualidade, com produtividade 
adequada para suprir as necessidades do produtor e do mercado consumidor.

Estudos sobre investimentos financeiros em projetos de reflorestamentos que contemplem informações sobre custos de implantação, manutenção e comercialização da produção de madeira originada de florestas plantadas ainda é incipiente (SANGUINO, 2009).

No Estado da Paraíba, a atividade da eucaliptocultura ainda está em fase experimental com a maioria dos plantios realizados por pequenos proprietários de terra que destinam parte da terra de seus imóveis ao desenvolvimento da atividade pelo não uso da mesma e pela falta de conhecimento acabam por não conduzir o plantio da forma adequada, acarretando assim em prejuízos ao final do ciclo.

Assim, conhecer a viabilidade econômica de projetos florestais é imprescindível para o estabelecimento da atividade, uma vez que, é fortemente afetada pelo horizonte de tempo, os custos de produção, o preço da madeira a produtividade do local e a taxa de desconto (NOGUEIRA FILHO et al., 2017).

Visando avaliar projetos florestais, além da produtividade, recomenda-se adotar critérios de avaliação econômica que consideram a variação do capital no tempo, como: Valor Presente Líquido (VPL), Taxa Interna de Retorno (TIR), Razão Benefício/Custo (B/C). Segundo Rezende, Oliveira (2013) esses métodos consideram o valor do capital no tempo, ou seja, atribuem diferentes ponderações às receitas líquidas em função de sua distribuição ao longo do tempo, sendo os mais indicados para a análise de projetos no setor florestal.

Diante disso, o objetivo deste trabalho é avaliar a viabilidade econômica e a produtividade de um povoamento de Eucalyptus spp. mal conduzido no Estado da Paraíba em relação à um povoamento em condições hipoteticamente adequadas de manejo e produtividade.

\section{Material e Métodos}

O estudo foi conduzido em um povoamento de Eucalyptus spp., com idade de 10 anos e área de 43,7 hectares, localizado na região metropolitana de João Pessoa, Paraíba. O clima da região, segundo a classificação de Köppen, é do tipo As, tropical com estação seca de verão, apresentando temperatura média de $25,4^{\circ} \mathrm{C}$ e precipitação anual de $1436 \mathrm{~mm}$ (ALVARES et al., 2013).

$\mathrm{Na}$ ocasião do inventário florestal, as árvores foram encontradas com espaçamento inicial de $1,5 \mathrm{x}$ $3,0 \mathrm{~m}$, sendo alocadas 20 parcelas de forma aleatória e com dimensão de 15 x $15 \mathrm{~m}\left(225 \mathrm{~m}^{2}\right)$ cada, sendo proporcional ao espaçamento de plantio, perfazendo uma área amostral de $4500 \mathrm{~m}^{2}$. Foram obtidas a circunferência a altura do peito (CAP) e a altura total das árvores presentes no interior da parcela que apresentaram CAP $\geq 10 \mathrm{~cm}$.

Para a estimativa volumétrica a ser obtida com a exploração do povoamento, foi considerado o limite máximo de confiabilidade admitido de $20 \%$ de erro a $95 \%$ de probabilidade. Devido à grande mortalidade de árvores encontrada no povoamento (cerca de 80\%), as mesmas foram divididas em árvores vivas e árvores mortas em pé para obtenção das estimativas volumétricas, uma vez que, embora mortas, as árvores apresentaram rendimento lenhoso passível de comercialização. A partir de então foi feita a quantificação das árvores e calculado seu volume individual a partir da multiplicação de sua área basimétrica, altura total e fator de forma comumente utilizado para a espécie ( $\mathrm{ff}=0,5$ ). Foi adotado esse método em virtude da inexistência de equações volumétricas desenvolvidas para Eucalyptus spp. na região do presente estudo.

Com a obtenção dos dados assumiu-se dois cenários para verificar a viabilidade econômica do povoamento, sendo: Cenário 1 - o povoamento atual, aquele em que houve baixo investimento de implantação e manutenção inicial, ocasionando uma alta taxa de mortalidade de árvores (Figura 1); e o Cenário 2 - o povoamento esperado (simulado), ou seja, aquele em que houve maior investimento para a implantação e manutenção em todas as fases de desenvolvimento, visando a otimização de todos os recursos disponíveis e resultando, assim, em um povoamento com produtividade aceitável para a região de estudo (em média $35 \mathrm{~m}^{3} \cdot \mathrm{ha}^{-1} \cdot \mathrm{ano}^{-1}$ ).

\section{REGRAS GERAIS}

\section{Figuras e gráficos}

Os títulos das figuras e tabelas devem ser autoexplicativos (somente em português ou inglês em caso de artigos neste idioma) e sua formatação conforme apresentado na Figura 1 e Tabela 1. As dimensões em ambos os casos, não devem exceder $16 \mathrm{~cm}$ em largura, e devem ser inclusos sempre com a orientação da página na forma retrato.

As figuras e tabelas devem ser numeradas sequencialmente, com algarismo arábico, e apresentadas logo após a sua citação no texto. As chamadas podem ser no início ou fim da frase entre parênteses. Por exemplo: Na Figura 1, observa-se... (Figura 1). 


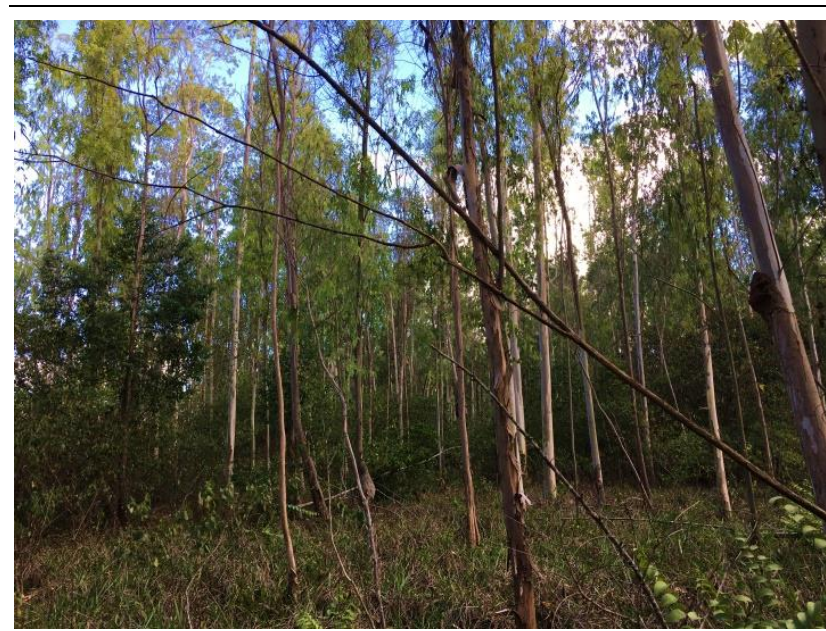

Figura 1 - Aspecto do povoamento de Eucalyptus spp. ma conduzido na Paraíba.

Para a comparação da viabilidade dos cenários foram utilizados os seguintes indicadores econômicos: Valor Presente Líquido (VPL), Taxa Interna de Retorno (TIR) e Razão Benefício/Custo $(B / C)$, calculados com base no fluxo de caixa de cada cenário (10 anos), utilizando-se a Taxa Mínima de Atratividade (TMA) de 6,5\% ao ano, baseada na Taxa SELIC do mês de março de 2019. O horizonte de planejamento de 10 anos foi utilizado devido à idade atual em que o povoamento se encontra.

A viabilidade econômica pelo método do VPL, de acordo com Rezende, Oliveira (2013), é indicada pela diferença positiva entre receitas e custos, atualizados de acordo com determinada taxa de desconto (Equação 1). Quanto maior o VPL, mais atrativo será o projeto. A Taxa Interna de Retorno de um projeto é a taxa anual de retorno do capital investido, tendo a propriedade de ser a taxa de desconto que iguala o valor atual das receitas ao valor atual dos custos do projeto (Equação 2). A razão Benefício/Custo estabelece a relação entre o valor atual das receitas e o valor atual dos custos. (Equação 3). Quando a razão B/C>1, o VPL é maior que 0 e a TIR é maior que a taxa do projeto.

$$
\begin{gathered}
V P L=\sum_{j=0}^{n} R_{j}(1+i)^{-j}-\sum_{j=0}^{n} C_{j}(1+i)^{-j}(\text { Equação } 1) \\
\sum_{j=0}^{n} R_{j}(1+T I R)^{-j}=\sum_{j=0}^{n} C_{j}(1+T I R)^{-j}(\text { Equação } 2) \\
B / C=\frac{\sum_{j=0}^{n} R_{j}(1+i)^{-j}}{\sum_{j=0}^{n} C_{j}(1+i)^{-j}}(\text { Equação } 3)
\end{gathered}
$$

em que: $R_{j}=$ receita no período $j ; C_{j}=$ custo no período $j ; i=$ taxa de juros; $j=$ período de ocorrência da receita ou do custo $(0 \ldots \mathrm{n}) ; \mathrm{n}$ = número máximo de períodos de duração do projeto.

A estimativa dos custos do povoamento atual (Cenário 1) foi obtido com o administrador da Fazenda, sendo consideradas para a implantação despesas de preparo do solo, combate a pragas, controle de ervas daninhas, aquisição de mudas, insumos (adubos, herbicidas e pesticidas) e mão de obra. Para a manutenção foram considerados apenas despesas com controle de mato-competição.

Para o Cenário 2 foram obtidas na literatura as despesas médias para a implantação e condução de um povoamento de Eucalyptus spp., considerando um maior investimento para se conseguir a máxima produtividade para as condições da região. Para a implantação considerou-se as mesmas despesas para o Cenário 1, porém, com maior tecnologia de preparo de solo e aquisição de mudas clonais. Já em relação às manutenções, no $1^{\circ}$ e $2^{\circ}$ ano foram consideradas as despesas com capina química mecanizada, adubação de cobertura, combate a pragas e manutenção de aceiros e carreadores. A partir do $3^{\circ}$ ano foram consideradas apenas as despesas com manutenção de aceiros e carreadores, além de repasses para controle de pragas.

A receita foi calculada com base na venda da madeira em pé no ciclo de 10 anos para ambos os Cenários. Para tanto, considerou-se a venda da madeira para energia (lenha) de $\mathrm{R} \$ 45,00 / \mathrm{m}^{3}$, sendo o valor médio encontrado na região do presente estudo.

Os dados foram processados com auxílio de planilha eletrônica do Microsoft Excel 2010 ®.

\section{Resultados e Discussões}

O povoamento atual (Cenário 1) apresentou uma alta taxa de mortalidade (cerca de 80\%), o que corresponde a um déficit de 938 árvores para um povoamento conduzido em condições ideais, admitindo-se mortalidade natural de $10 \%$ e

\begin{tabular}{|c|c|c|c|c|}
\hline Cenários & $\begin{array}{l}\text { No Árv./ha } \\
\text { (10 anos) }\end{array}$ & $\begin{array}{l}\text { Prod. } \\
\left(\mathbf{m}^{3} / \mathbf{h a}\right)\end{array}$ & $\underset{\left(\mathbf{m}^{3} / \mathbf{h a} / \mathbf{a n o}\right)}{\text { IMA }}$ & $\begin{array}{c}\text { Rentabilidade considerando o valor do } \\
\text { m }^{3} \text { da lenha de eucaliptos para fins } \\
\text { energéticos de } R \$ 45,00 / m^{3}(R \$ / h a)\end{array}$ \\
\hline \multirow{2}{*}{$\begin{array}{c}\text { Cenário1 } \\
\text { (Povoamento atual) }\end{array}$} & 462 vivas & 130,5 & 13,05 & \multirow{2}{*}{$\mathrm{R} \$ 7.263,00^{* *}$} \\
\hline & 367 mortas em pé & 30,9 & & \\
\hline $\begin{array}{c}\text { Cenário } 2 \\
\text { (Povoamento esperado) }\end{array}$ & $1400^{*}$ & 350 & 35 & $\mathrm{R} \$ 15.7500,00$ \\
\hline Perda (diferença) & -938 & $-219,5$ & $-21,95$ & - R\$ 8.487,00 \\
\hline
\end{tabular}
realização de desbaste aos 7 anos (Tabela 1).

Tabela 1. Produtividade e rentabilidade do povoamento de Eucalyptus spp. para o Cenário 1 e Cenário 2. 
* Considerando-se espaçamento inicial de plantio de 1,5 m x 3,0; $10 \%$ de mortalidade natural e redução de $30 \%$ da densidade aos 7 anos (desbaste);

** Considerando-se a venda da madeira das árvores vivas e mortas em pé.

Para obtenção do Incremento Médio Anual (IMA) do Cenário 2 (povoamento esperado), foi utilizado $35 \mathrm{~m}^{3} \cdot \mathrm{ha}^{-1} \cdot \mathrm{ano}^{-1}$, sendo, segundo Floriano (2018), a média de incremento utilizada para povoamentos de eucalipto. Em Alagoas, há uma projeção de se obter $45 \mathrm{~m}^{3} \cdot \mathrm{ha}^{-1} \cdot$ ano $^{-1}$ de madeira de Eucalyptus sp. a partir de clones (FLORIANO, 2018). Algumas empresas, porém, já atingem os 60 $\mathrm{m}^{3} \cdot \mathrm{ha}^{-1} \cdot$ ano $^{-1}$ em rotações de 7 anos de eucalipto (SNIF, 2018). Esses dados reforçam a baixa produtividade do povoamento de eucaliptos em estudo, o qual apresentou IMA de 13,05 $\mathrm{m}^{3} \cdot \mathrm{ha}^{-1}$.ano 1 , influenciado significativamente pelo baixo investimento na ocasião de implantação e falta de manutenções periódicas, ocasionando a alta mortalidade de árvores.

Com isso, tem-se que a colheita florestal que deveria resultar em $350 \mathrm{~m}^{3} \cdot \mathrm{ha}^{-1}$ de lenha de eucaliptos estimado pelo Cenário 2, produzirá apenas 161,4 $\mathrm{m}^{3} \cdot \mathrm{ha}^{-1}$ (Cenário 1), acarretando em uma perda de aproximadamente $54 \%$ da capacidade de produção madeireira. As estimativas do inventário florestal apresentaram um erro de amostragem $14,9 \%$ a $95 \%$ de probabilidade de confiança para a média volumétrica.

Para decisão da atividade é imprescindível conhecer o valor comercial da lenha. No Estado da Paraíba, $1 \mathrm{~m}^{3}$ de lenha comercializada em pé na floresta custa em média $\mathrm{R} \$ 45,00$, sem serem considerados os custos de colheita e transporte. Assim, considerando o déficit na produtividade para o povoamento mal conduzido (Cenário 1), a receita deixou de gerar $\mathrm{R} \$$ 8.487,00 .ha $^{-1}$ quando comparado ao valor de rentabilidade para o povoamento esperado (Cenário 2).

Ainda de acordo com a análise econômica observou-se que os custos de implantação do povoamento de Eucalyptus spp. foram ainda maiores do que os custos de manutenção, como também observado por Virgens et al. (2016). Porém, apesar do Cenário 1 ter menor investimento, acarretando em menor despesa de implantação e manutenção, se comparado com o Cenário 2, que teve maior investimento e despesa, ambas atingiram êxito ao fim do ciclo de 10 anos, pois as despesas foram diretamente proporcionais as receitas (Tabela 2).

Tabela 2. Análise econômica para o Cenário 1 (Povoamento atual) e Cenário 2 (Povoamento esperado) nos 10 anos de idade avaliados.

\begin{tabular}{|c|c|c|c|c|c|}
\hline \multirow{2}{*}{ Ano } & \multirow{2}{*}{ Atividade } & \multicolumn{2}{|c|}{ Cenário 1 (Povoamento Atual) } & \multicolumn{2}{|c|}{ Cenário 2 (Povoamento Esperado) } \\
\hline & & Despesas/ha & Receitas/ha & Despesas/ha & Receitas/ha \\
\hline 0 & Implantação & $\mathrm{R} \$ 1.900,00$ & - & $\mathrm{R} \$ 3.200,00$ & - \\
\hline 1 & Manutenção 1 & $\mathrm{R} \$ 200,00$ & - & $\mathrm{R} \$ 400,00$ & - \\
\hline 2 & Manutenção 2 & $\mathrm{R} \$ 200,00$ & - & $\mathrm{R} \$ 400,00$ & - \\
\hline 3 & Manutenção 3 & - & - & $\mathrm{R} \$ 200,00$ & - \\
\hline 4 & Manutenção 4 & - & - & $\mathrm{R} \$ 200,00$ & - \\
\hline 5 & Manutenção 5 & - & - & $\mathrm{R} \$ 200,00$ & - \\
\hline 6 & Manutenção 6 & - & - & $\mathrm{R} \$ 200,00$ & - \\
\hline 7 & Manutenção 7 & - & - & $\mathrm{R} \$ 200,00$ & $\mathrm{R} \$ 3.307,50$ \\
\hline 8 & Manutenção 8 & - & - & $\mathrm{R} \$ 200,00$ & - \\
\hline 9 & Manutenção 9 & - & - & $\mathrm{R} \$ 200,00$ & - \\
\hline \multirow[t]{5}{*}{10} & Colheita & - & $\mathrm{R} \$ 7.263,00$ & - & $\mathrm{R} \$ 12.442,50$ \\
\hline & VPL (R\$/ha) & \multicolumn{2}{|c|}{ R\$ 1.605,06 } & \multicolumn{2}{|c|}{$\mathbf{R} \$ \mathbf{3 . 8 6 1 , 4 9}$} \\
\hline & VPL $(R \$ / 43,7$ ha $)$ & \multicolumn{2}{|c|}{$\mathrm{R} \$ \mathbf{7 0 . 1 4 1 , 2 9}$} & \multicolumn{2}{|c|}{ R\$ $168.747,30$} \\
\hline & TIR & \multicolumn{2}{|c|}{$12,50 \%$} & \multicolumn{2}{|c|}{$14,49 \%$} \\
\hline & $\mathrm{B} / \mathrm{C}$ & \multicolumn{2}{|c|}{1,71} & \multicolumn{2}{|c|}{1,79} \\
\hline
\end{tabular}

O cenário 2 que mais investiu teve maior despesa, porém, gerou uma receita muito superior ao Cenário 1 se considerarmos a rentabilidade total do povoamento florestal.

Nogueira Filho (2017) estudando um povoamento de eucaliptos para destinação moveleira em Marco, Ceará, também comparou a rentabilidade da atividade com e sem investimento para implantação do povoamento. Os autores verificaram que para um povoamento houve uma maior quantidade e qualidade de tratos silviculturais e para o outro não, resultando em uma maior produtividade para aquele em que houve maior investimento, corroborando com os resultados do presente estudo.

O Valor Presente Líquido (VPL), calculado de acordo com a Taxa Mínima de Atratibilidade (TMA) de $6,5 \%$ foi positivo para este estudo tanto no Cenário 1 com R\$1.605,06.ha ${ }^{-1}$, como no Cenário 2 com R\$ 3.861,49. $\mathrm{ha}^{-1}$. Resultados semelhantes também foram observados por Nogueira Filho (2017), que utilizou a TMA de $6,38 \%$ avaliando o povoamento de eucaliptos para movelaria com e sem investimento.

Em estudo realizado por Queiroz, Silva (2016), ao avaliar os aspectos econômicos de um 
povoamento de Eucalyptus spp. para lenha na região do baixo Acre, considerando a venda da madeira com e sem o custo da terra, foram encontrados VPL's negativos utilizando diferentes TMA's $(6 \%$, $8 \%, 10 \%$ e $12 \%$ ), apontando a inviabilidade da atividade para a região em questão, devido ao alto custo de produção ou até mesmo do baixo valor de mercado para comercialização da lenha.

No presente estudo não foi contabilizado o valor da terra, considerando que no Estado da Paraíba ainda não há perspectivas de aquisição imobiliária para implantação da atividade, geralmente sendo realizadas por pequenos produtores que destinam áreas em desuso, ou seja, que já não geram receita. Caso venha surgir especulação imobiliária para isso, novos cálculos devem ser incrementados para analisar a viabilidade de projetos florestais, como por exemplo, o Valor Esperado da Terra (VET). Em um estuda da viabilidade econômica quanto à produção de eucaliptos para fins energéticos no Paraná, Goltz (2013) verificou que, quando considerada a compra da terra para o desenvolvimento da atividade, o VPL foi negativo e a TIR foi inferior ao custo de oportunidade do capital.

Para o presente estudo pode-se observar que mesmo diante do baixo investimento/custo realizado no Cenário 1, ainda assim, a atividade da Eucaliptocultura foi viável de acordo com as Taxas Internas de Retorno (TIR) obtidas, sendo superiores a TMA de $6,5 \%$. Esse fato aponta a viabilidade e potencialidade da atividade da Eucaliptocultura no Estado da Paraíba. O resultado corrobora com Rezende, Oliveira (2013) e Silva et al. (2005), que reforçam que o TIR superior ao TMA assegura a viabilidade do projeto para o indicador em análise.

No Cenário 2 (povoamento esperado), considerando a realização de um desbaste aos 7 anos de idade no povoamento, resultaria em duas receitas no fluxo de caixa, contribuindo assim para um maior êxito em sua receita final e consequentemente maior contribuição na taxa de retorno (TIR=14,49\%). Sendo assim, o Cenário 2 apresenta-se mais viável para a implantação de projetos de eucaliptocultura no Estado da Paraíba.

A Razão Benefício/Custo (B/C) também ressalta essa viabilidade, com 1,71 para o povoamento atual (Cenário 1) e 1,79 para o povoamento esperado (Cenário 2). Apesar da baixa produtividade verificada no povoamento mal conduzido, ainda assim, os valores investidos ao final do ciclo de 10 anos para os dois cenários se aproximaram, sendo ainda maior para o povoamento com produtividade esperada (Cenário 2).

A relação $\mathrm{B} / \mathrm{C}$ encontrada para o presente estudo em ambos os Cenários foram superiores ao encontrado por Virgens et al. (2016) de 1,44, que avaliou TMA's de $8 \%, 10 \%$ e $12 \%$ em um povoamento de eucaliptos no Sudoeste da Bahia, Estado conhecido por extensos povoamentos comerciais de eucaliptos.

Merece destaque ainda que ao avaliar o VPL para toda a área do povoamento (43,7 ha), foi encontrada uma grande diferença entre os VPL's, sendo R\$ $70.141,29$ para o povoamento atual (Cenário 1 ) e $\mathrm{R} \$$ 168.747,30 para o povoamento esperado (Cenário 2), ou seja, há uma perda de R $\$ 98.606,00$ que pode estar relacionado a má condução do povoamento. $\mathrm{O}$ resultado enfatiza a necessidade de se avaliar mais de um único indicador econômico, para maior segurança do investidor, pois se fosse avaliado apenas o indicador Razão benefício/Custo (B/C) poderia ser concluído que não há diferença rentável entre os povoamentos, já que a diferença entre ambos foi de apenas 0,08 .

\section{Conclusões}

A atividade da eucaliptocultura no Estado da Paraíba mostrou-se economicamente viável apresentando VPL positivo, TIR maior que a TMA e $\mathrm{B} / \mathrm{C}>1$ para ambos os cenários avaliados.

Para o estudo em específico pode se recomendar a substituição do povoamento a fim de que seja reconduzido de forma adequada, com investimentos necessários de modo a assegurar o máximo retorno comercial.

Estudos sobre o manejo de Eucaliptos no estado da Paraíba merecem atenção, a fim de que sejam definidos os ciclos de corte ideais de modo a favorecer a produtividade madeireira da região, diminuindo assim a pressão existente sobre a flora nativa para demanda energética.

\section{Referências}

ALVARES, C. A.; STAPE, J. L.; SENTELHAS, P. C.; GONÇALVES, J. L. M.; SPAROVEK, G. Köppen's climate classification map for Brazil. Meteorologische Zeitschrift, v. 22, n. 6, p. 711-728, 2013.

FloRIANO, E. P. Potencialidades de Produção Florestal em Alagoas. Rio Largo: Edição do autor, 2018. $182 \mathrm{p}$.

GOLTZ, V. Viabilidade econômica para a implantação de cultivo de eucalipto para fins energéticos na Seara Indústria e Comércio de Produtos Agropecuários LTDA. 2013. 38 f. Dissertação (Mestrado em Ciência e Tecnologia de Sementes) - Programa de Pós-Graduação em Ciência e Tecnologia de Sementes, Universidade Federal de Pelotas, Pelotas.

MOLEDO, J. C.; SAAD, A. R.; DALMAS, F. B.; ARRUDA, R. O. M.; CASADO, F. Impactos ambientais relativos à silvicultura de eucalipto: uma análise 
comparativa do desenvolvimento e aplicação no plano de manejo florestal. Geociências. São Paulo, v. 35, n. 4, p. 512-530, 2016.

NOGUEIRA FILHO, F. P.; BAJAY, M. M.; SOUSA, J. A.; ARAÚJO, J. D. M.; CORREIA, D. Viabilidade econômica da produção de eucalipto no polo moveleiro de Marco - Ceará. Revista iPecege, v. 3, n. 4, p. 22-34, 2017.

QUEIROZ, A. M.; SILVA, Z. A. G. P. G. Aspectos econômicos dos plantios com eucalipto (Eucalyptus spp.) na região do Baixo Acre. Floresta, Curitiba, PR, v. 46, n. 3, p. 287 - 296, 2016.

REZENDE, J. L. P.; PADUA, C. T. J.; OLIVEIRA, A. D.; SCOLFORO, J. R. S. Análise econômica de fomento florestal com eucalipto no Estado de Minas Gerais. Cerne, Lavras, v. 12, n. 3, p. 221-231, 2006.
REZENDE, J. L. P.; OLIVEIRA, A. D. Análise econômica e social de projetos florestais. 3. Ed. Rev. Ampl. Viçosa, MG: Ed. UFV; 2013. 385 p.

SILVA, M. L.; JACOVINE, L. A. G.; VALVERDE, S. R. Economia florestal. 2. Ed. Viçosa: UFV; 2005.

SANGUINO, A. C. Custos de implantação e rentabilidade econômica de povoamentos florestais com Teca no Estado do Pará. Ciências Agrárias, v. 52, p. 61-78. 2009.

VIRGENS, A. P.; FREITAS, L. C.; LEITE, A. M. P. Análise Econômica e de Sensibilidade em um Povoamento Implantado no Sudoeste da Bahia. Floresta e Ambiente, v. 23, n. 2, Seropédica, 2016. 